\title{
Sound Quality Enhancement by using the Single Core Exciter in OLED Panel
}

\author{
Sungtae Lee ${ }^{1}$, Kwanho Park ${ }^{1}$, and Hyungwoo Park ${ }^{2 * *}$, \\ ${ }_{1}^{1}$ LG Display, Paju-si, Gyunggi-do, Republic of Korea \\ ${ }^{2}$ School of Information Technology, Soongsil University, Seoul, Republic of Korea \\ [e-mail: pphw@ssu.ac.kr] \\ Corresponding author: Hyungwoo Park
}

Received Septebmer 10, 2019; revised November 27, 2019; revised January 22, 2020; accepted February 6, 2020; published February 29, 2020

\begin{abstract}
With the development of display engineering and technology, the screen and sound quality of information devices such as TVs are improving. The screen used LEDs via LCD and PDP and a large flat panel in the early CRT to create super-high resolution. The sound is improved by directly vibrating a thin and simple panel, such as an OLED. In our previous study, the exciter speaker was attached to the rear of the OLED panel to be used as the diaphragm of the speaker, and the sound quality was as good as that of the TV using the existing dynamic speaker. This method supplied the viewer with the direct sound coming from the panel, delivering clear sound, and the sound and image came from the same location, thus giving the viewer high immersion and maximizing the effect of information transfer. OLED exciter speakers, however, have a special directivity, which tends to slightly attenuate the tone at the very center of the screen. This study improves the sound quality by improving the structure of the exciter speaker and the radiated sound of the flat panel display. A 2-in-1 Exciter is made into a single core to improve the speaker's radiation pattern.
\end{abstract}

Keywords: OLED Panel Speaker, Exciter Speaker, Flat Panel Speaker, Sound Quality Enhancement, Sound Radiation 


\section{Introduction}

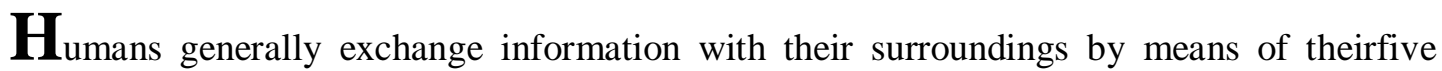
sensory organs. Among the five senses, it is usually seeing and hearing that confer understanding. In addition, they communicate most of the information from the time of birth by means of repeated learning. Visual and auditory information is not only delivered quickly and accurately but also conveys a variety of cultural, emotional, and health information and contents along with linguistic and figurative meanings. IT technology has developed rapidly since the 20th century, the speed of digital computing has been improved, and storage memory has been increased to develop various information devices. Among them, the most influential and diverse information is transmitted to modern people on the TV. Early televisions were developed to deliver video and sound from far away. The cathode tube was used to present the image, and the image quality was poor because of the bulky and limited technology. Users were more dissatisfied with image noise, low quality, low resolution, and screen noise than with with sound quality. A color image is spread in CRT, mono sound improves the quality of a dynamic speaker, and two channels gradually improve viewers' satisfaction. By means of the development of display technology and materials, image quality has been improved by PDP and LCD. In particular, by increasing the pixels and adjusting the resolution, the resolution has been improved from SD to FHD, UHD, and recently 8K. In order to improve viewers' satisfaction by improving image quality and the design of the screen, the bezel, other than the portion where the image is displayed, is minimized. Because of this change in appearance, the speakers placed on the front moved behind the screen. As a result, viewers can hear the sound that is reflected from the back or the wall and is affected by the furniture layout and materials (acoustic reflection characteristics). Although it has been developed into an $8 \mathrm{~K}$ resolution LED TV, which is the ultimate picture quality, the sound, emphasized by this phenomenon and its disadvantages, is unclear and of poor quality. In particular, the sense of immersion is reduced because of the mismatch of senses (audio and video) trained since childhood, and information transmission by sound is inefficient [1-6].

The development of display technology and information and communication technology has enabled creation of 3D images, ultra-high-definition images, and image that can feel virtual. Along with these developments, the two left and right channels are also transmitting a more significant number of signals to form a three-dimensional effect. However, in a general TV, only two channels, the left and right speakers, are used to transmit sound, and external devices, such a as sound-bar and home theater are used for high-quality sound. In particular, in modern flat-panel TVs, the sound source of the speaker mounted on the back of a large screen is affected by the surrounding environment, causing loss and distortion in the sound transmission path. Various excitation sources generate sound, and the vibration of air molecules stimulates human auditory organs by means of the transfer of wave motion. Since this sound is transmitted in wave motion, there are refraction, reflection, diffraction, and so on, and because sound is invisible to the human eye, it is determined by the quality of the final listening point rather than by direct tracking of the location of origin or transmission path. Because the sound is delivered directly to the brain by means of the auditory organ, there is an advantage that can easily stimulate the imagination by means of the linkage of the cerebral tools to produce a rich effect with little change. For this reason, previous research was conducted in which the viewing effect was improved when the point where the sound is generated and the moving point of the image coincide [2][7-9]. 
Because of the refraction and diffraction of sound, modern flat-panel TVs hide the speakers behind the screen. However, the speakers behind the screen can transmit sound, but the sound quality is affected by the environment and surroundings. Viewers watch to increase the sound pressure to compensate for unintended degradation. Loud sound can be viewed as a relatively higher SNR for the viewer, but this loudness is inconvenient for those who are not watching. To overcome this drawback, we studied the exciter speaker technology using an OLED panel as the diaphragm of the speaker. In our previous research, we introduced the OLED panel speaker and the method of composing the stereo sound of the two left and right channels [10][11]. In addition, two oscillators simultaneously moved to improve the sound quality and sound pressure of this exciter speaker. As a result, the two cores interfere with each other during operation [11][12] the acoustic characteristics were affected at 30 degrees from the front of the OLED panel speaker, and the directivity characteristics deteriorated at high frequencies above $8 \mathrm{kHz}$. This study introduces a way to compensate for these shortcomings and to improve sound quality. We developed a single-core exciter that overcomes low sound pressure and eliminates mutual interference in order to improve sound quality, by overcoming the narrow directional characteristics, which are a disadvantage of OLED panel speakers.

\section{Previous research and principal background}

\subsection{Dynamic speaker structure and panel exciter speaker structure}

The ordinary loudspeaker was invented in the early twentieth century, with a principle and technology used to make sound continuously without significant change. The dynamic speaker is divided into three parts: the vibration system, the support system, and the magnetic system. The support system consists of a gasket that plays the role of packing the speaker, and the other with the frame fixed to the frame, and an edge that acts as a body that fixes the vibration system and the magnetic system to the frame. The vibration system connects the mechanical vibration of the voice coil to the diaphragm, which creates the pressure wave (long wave) of the air; the edge, damper, etc., support the vibration structure and make the movement smooth. The magnetic system is composed of plate, magnet, and yoke that form the magnetic flux generated by the magnet on the magnetic gap. For nearly 100 years, dynamic speakers have been widely used to produce sound evenly from low to high frequencies. Although the development of materials and of fabrication techniques have progressed, the main principles and forms remain unchanged. Fig. 1 shows the cross-sectional structure of a dynamic speaker [9][11].

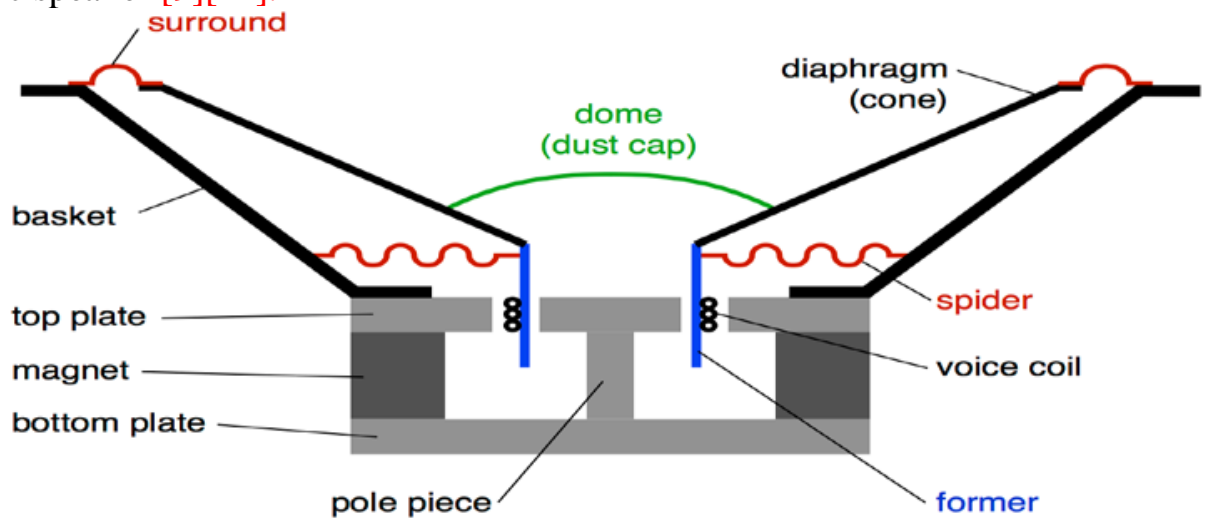

Fig. 1. Simplified model of dynamic loudspeaker [11] 
The structure of the OLED panel vibration speaker is shown in Fig. 2. The exciter was developed by thinning the magnetic field by eliminating the vibration plate parts of the diaphragm, cap, and edge part from the dynamic speaker structure. When the exciter is attached directly to an OLED panel and transmits vibration, the panel becomes the diaphragm of the dynamic speaker and produces sound. The structure shown in Fig. $\mathbf{2}$ is the same as the structure of the exciter, which is applied to the OLED panel speaker. The support and magnetic system are the same asin the structure of the dynamic speaker. Part of the diaphragm is replaced by a panel. In particular, the part that acts as the edge of the dynamic speaker contains the vibration of the exciter and is called the enclosure. In dynamic speakers, diaphragms typically use paper that is light in mass and rigid. OLED panels have properties similar to metal plates, which are more elastic than paper, so vibration in OLED panels must be well controlled. Also, ordinary TV sound should consist of at least two stereo channels. Stereo channels are impossible unless the OLED panel is physically divided in two. Therefore, this problem was solved by forcibly controlling the physical vibration of the OLED panel between the two exciters. The enclosure was devised to control panel vibration. By means of this enclosure, one continuous OLED panel could be separated into two or more exciter channels, and channel crosstalk could be improved [2][6][10][12][13].

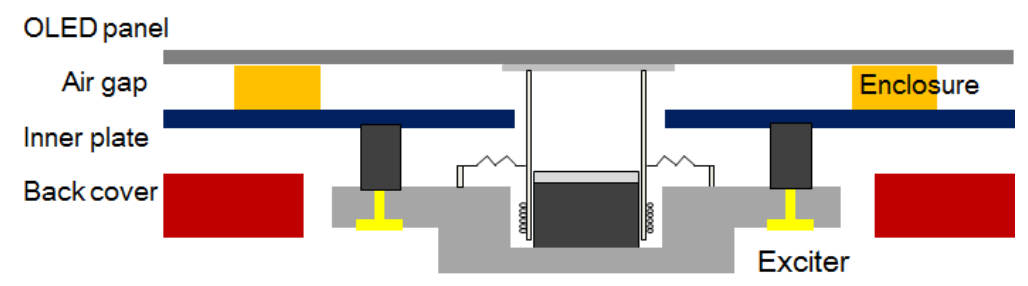

Fig. 2. A model of flat panel exciter speaker [6]

\subsection{The characteristic of OLED panel exciter speaker}

Fig. 3 shows the frequency response between the proposed OLED panel speaker and an ordinary flat-panel TV speaker. Red is the frequency response of the panel speaker used in the previous study, and black is the frequency response of the existing dynamic speaker system. It can be seen that the proposed method has a high-frequency response evenly over a broader range of existing methods. In particular, the response appears flat in the high-frequency band of $3 \mathrm{kHz}$ or more. In addition, it can be seen that the dip of the $500-\mathrm{Hz}$ band and the $2-\mathrm{kHz}$ band shown in the conventional method is reduced. Therefore, TVs with OLED panel speakers facing forward more than conventional TVs with existing speakers do not degrade the sound quality at high frequencies of $3 \mathrm{kHz}$ or more and exist up to the $20-\mathrm{kHz}$ high-frequency band [2][6][10][12].

The downward directional dynamic speaker unit applied to conventional TVs is designed and manufactured to reproduce sound evenly up to $20 \mathrm{~Hz}$. However, the analysis in the front center of the screen changes the acoustic value reflected by the speaker's directivity, the TV stand, or the surrounding environmental structure. Some modern TVs have corrective algorithms that improve the sound [14][15]. However, the OLED panel speaker is less affected by the environment in which the TV is installed, so it can use the good-quality state at the time of design and production. This feature improves intelligibility, especially in the frequency bands of sound/voice generated in TVs over conventional systems. Viewers experience faster 
understanding and realism in a clearer sound. Our previous study found that understanding and concentration is improved when the focus of the sound and the screen coincide [13][16].

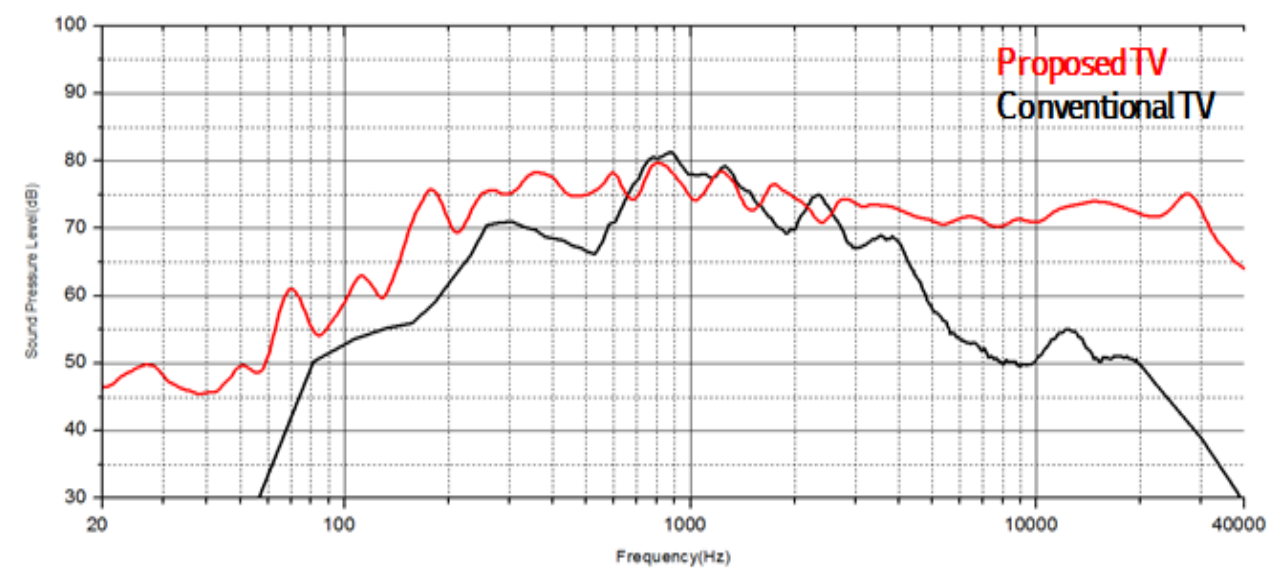

Fig. 3. Frequency Response Comparison between conventional TV and OLED Panel Speaker TV

In addition, the OLED panel exciter speaker can move the location of sound to the center or top of the screen. The result of shooting with sound camera SeeSV-S205 is shown in Fig. 4, in which, on a conventional TV, the sound is concentrated at the bottom of the screen. Take a closer look at the results. The center of the sound is reflected at the bottom of the screen. The proposed method can confirm that the sound is concentrated in the center of the screen, and it can be seen that the whole panel operates as the diaphragm of the big speaker. This is an advantage. in that the positions of the display and sound can coincide at the point where the screen speaks or plays. In addition, in view of the basic characteristics by which the sound is radiated and spread, the proposed method generates clear sound because it is delivered to the viewer as it is [13].
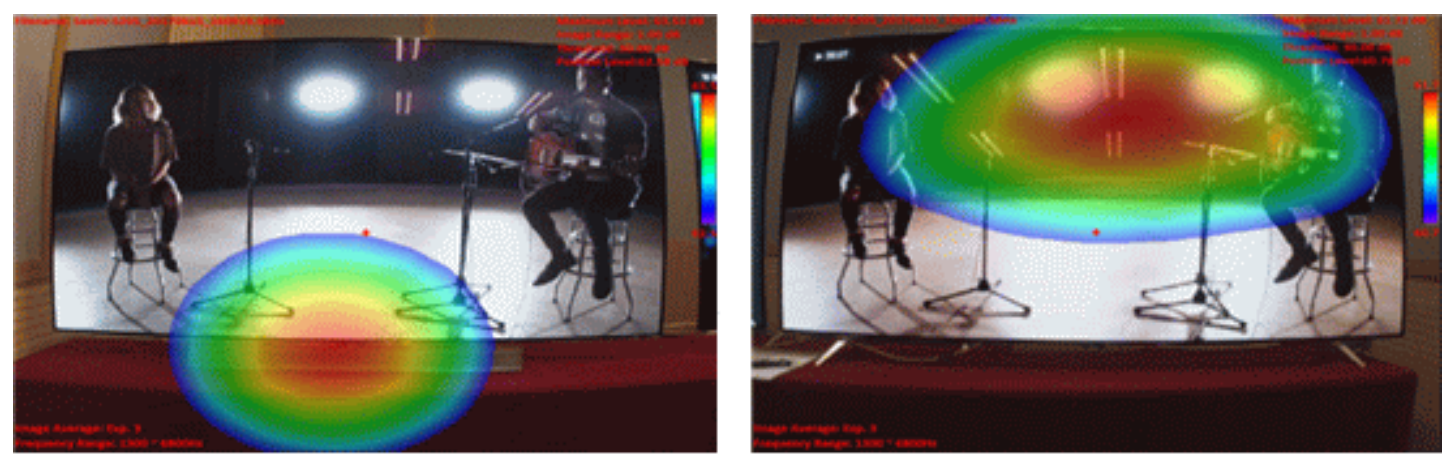

Fig. 4. Sound Image of conventional TV and proposed TV

\section{Improvement of Directional Sound Quality of Proposed Exciter Speaker}

\subsection{Dual-Core and Single-Core Exciter Speaker Designs}

In the previous study, the panel of OLED was made to vibrate to make sound, and sound quality was improved for commercialization of a TV using this exciter speaker. In the process, 
two exciters per channel were needed to produce more sound pressure that one exciter could produce, in order to have an optimal sound that parts can produce in a given space. As shown in Fig. 5 and Equation 1, producing a loud sound requires a strong electromagnetic signal or a strong magnetic field. As the thickness or size of the exciter increases for a strong magnet, the rear design must change. In the early stages of developing panel exciter speakers, two exciters were used simultaneously to overcome this limitation. That type of exciter could add an average volume of $3 \mathrm{~dB}$, making it possible to compose the sound field more smoothly than 72 $\mathrm{dB}$ when using a single exciter. However, two pole pieces were used, and the directivity was reduced. The sound pressure in the center also loses about $3 \mathrm{~dB}$, requiring improvement in volume and sound pressure [9][15].

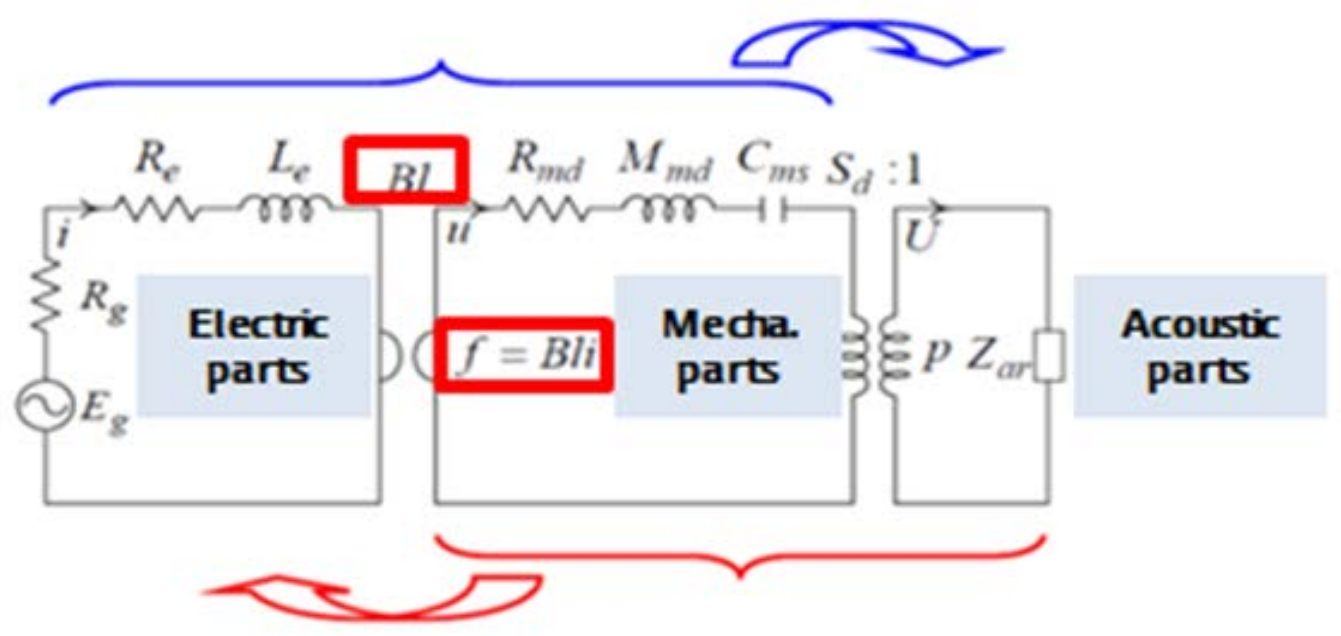

Fig. 5. Electro-Mechanical equal Model Block Diagram of Speaker [9][15]

Fig. 5 shows a block diagram of the sound produced by the electricomechanical equivalent model of the speaker. Equation 1 is an equivalent equation for converting the mechanical electrical parameters of the speaker into sound in Fig. 5 [9].

$S P L=94+20 \log \left(\frac{\rho \cdot S d \cdot B \cdot / \cdot V}{2 \cdot \pi \cdot M_{m s} \cdot \operatorname{Re}}\right)$

In order to maintain the same sound pressure, in the equivalent circuit and the formula, the OLED panel structure is the only way to increase the sound pressure by increasing the magnetic force. Mms is the effective mass, $\rho$ is air density, $V$ is voltage, $R e$ is DC resistance, a is acceleration, B is magnetic flux density, $\mathrm{l}$ is voice coil effective length, I is current, and Sd is effective diaphragm area. One way to improve the performance of the OLED panel speaker's output sound pressure is to strengthen the exciter's magnetic force. This method raises the magnetic force when applying the high magnetism of the permanent magnet. However, the yoke's thickness, the permanent magnet's thickness, and the pole piece's thickness do not increase the performance of permanent magnet linearly. If the threshold is exceeded, the performance indicator is saturated. Specifications indicating permanent magnet performance are indicated as $\mathrm{N} 48 \mathrm{H}$. Here, $\mathrm{N}$ means neodymium magnet and has strong magnetic properties in a small volume. Neodymium is easy to process and is widely used in a variety of products. 
The second number is grade, and the higher the number, the stronger the magnetic force in the same volume. The last $\mathrm{H}$ represents the allowable temperature. Neodymium gradually develops potatoes when used above acceptable temperatures. When $\mathrm{H}$ is used at 120 degrees or less, there is no problem [16-19].

In the early stage of development, the optimized $\mathrm{N} 48 \mathrm{H}$ magnet grade could be derived by means of the magnetic circuit simulation. $\mathrm{N} 48 \mathrm{H}$ was analyzed in the form of linearly increasing in magnetic force, but it was not applied, because it is the point where magnetic saturation starts, and the cost increases rapidly. Compared to a Circle Type 2-in-1, when the same grade of $\mathrm{N} 48 \mathrm{H}$ is used, the ellipse type is increased in the coil magnetic flux and total magnetic flux. We optimized the thickness of each component in the defined magnetic circuit thickness. The yoke thickness is $2.2 \mathrm{t}$, the magnet thickness is $4.0 \mathrm{t}$, the pole piece is $2.5 \mathrm{t}$, and the magnet flux is Max.

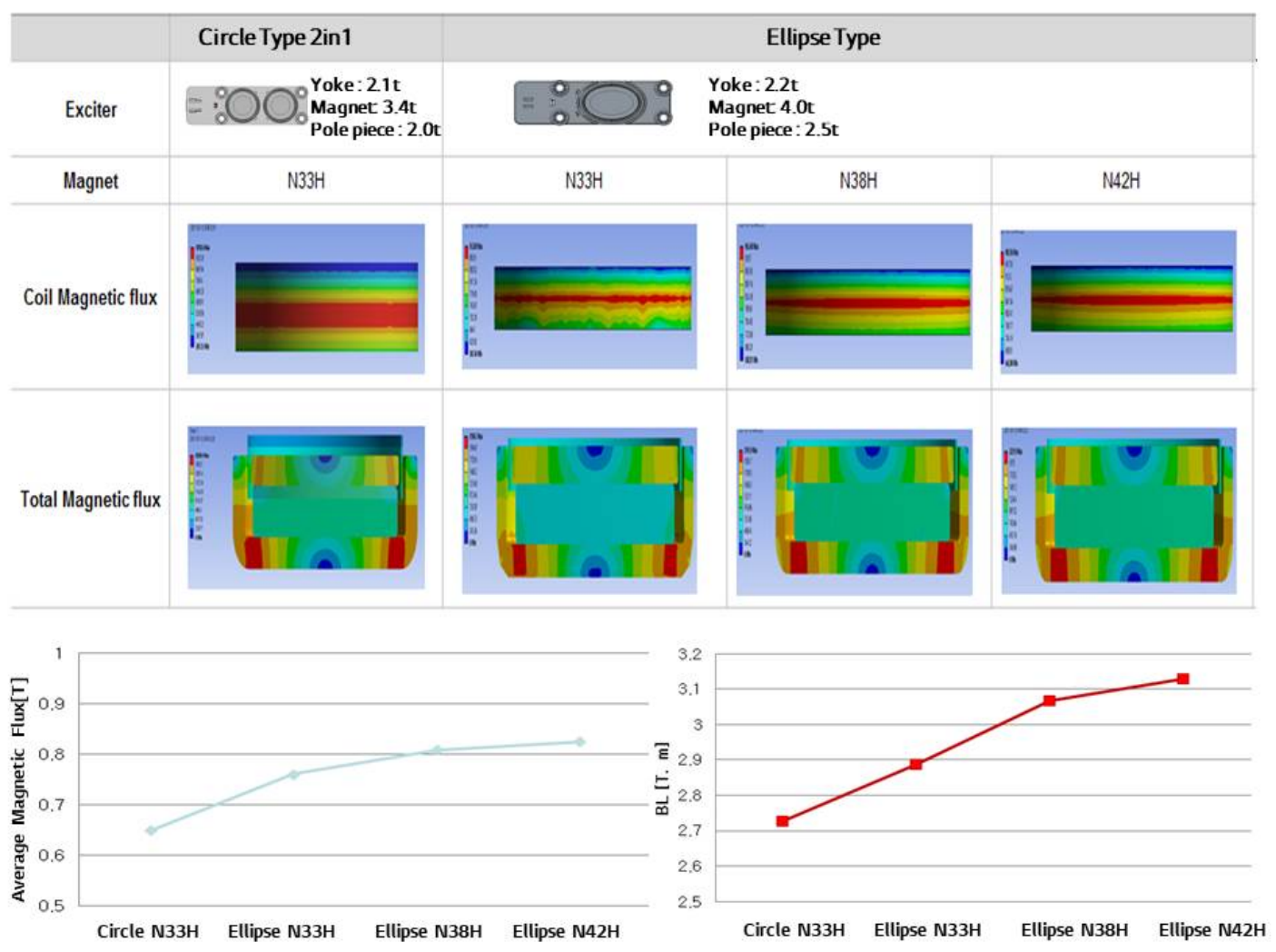

Fig. 6. Simulation results by magnet grade for magnetic system optimization

Fig. 7 shows the result of comparing the Circle Type Single and Dual Exciter applied with the same N33H magnet. It is confirmed that the sound pressure increases by $3 \mathrm{~dB}$ when a Dual Exciter is applied rather than a Single. Even if a Dual Exciter was used, the only sound pressure was increased without significant change in front. However, when the exciter of the same condition is doubled, the sound pressure of $6 \mathrm{~dB}$ should be increased. However, the $3 \mathrm{~d} B$ was improved because of the limitation of driving the system in the same phase. 

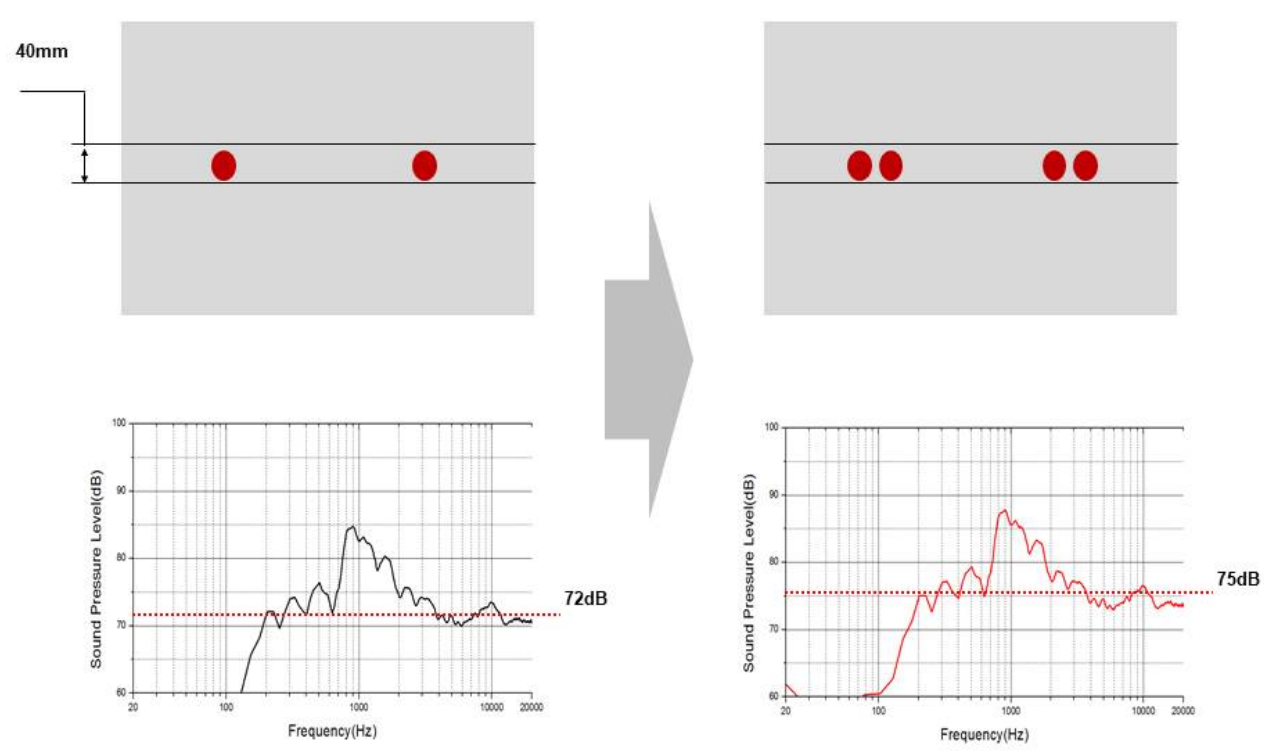

Fig. 7. N33H single and dual core frequency response vs. sound pressure

\subsection{Ellipse Type Single Core Exciter Acoustic Characteristics}

As a result of measuring the acoustic characteristics of the designed Ellipse-Type Single-Core Exciter, the overall sound pressure is improved by 1-3 dB in all frequency bands. The graph in Fig. 8 shows the result of the proposed Ellipse-Type Single-Core Exciter with the improved red line and the result of applying the 2-in-1 exciter to the black line. The measurement condition is $50 \mathrm{~cm}$ from the speaker in the anechoic chamber. The input signal is pink noise, and the input power is $1 \mathrm{~W}$. The results show that the ellipse-type single-core exciter appears high in the $300 \mathrm{~Hz}, 5 \mathrm{kHz}$, and $13 \mathrm{kHz}$ bands. In addition, it was confirmed that less ripple occurs in the overall response, resulting in less dip in the frequency response.

The acoustic hearing of the two types of exciters was also evaluated. These results are the difference in subjective assessment of two speaker type of the measurement results in Fig. 8. This evaluation was carried out according to the procedure proposed in [20]. A hearing assessment is the result of the same music under the same conditions [20]. Subjective listening evaluations differ in personal preferences. Accordingly, we collected 159 opinions, including the opinions of audio engineers with 20-30 years of experience. In the experiment, we listened directly to the two types of sounds and tested the results through a questionnaire. We evaluated the sound according to the exciter method installed on the TV from the front and the side. Participants watched the news, classical music, and pop music. The same contents were alternately watched for about 15 seconds, and a total of about 7 minutes of experiments were performed. The questions on the questionnaire were measured on the Likert-5 scale: sounds clear. Sounds good. Sounds accurate. Statistical analysis of the survey consisted of factor analysis, reliability analysis, and t-test analysis. 


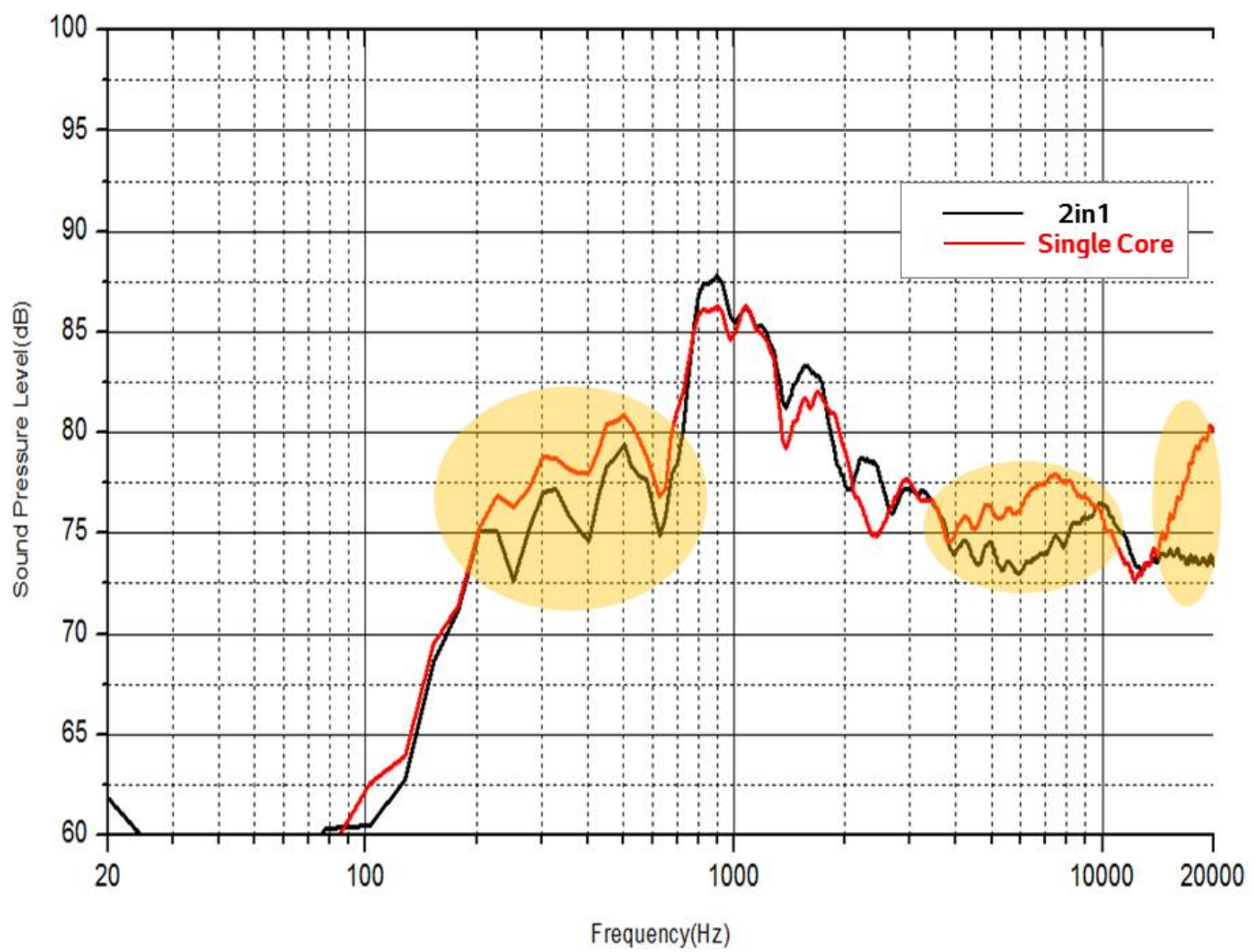

Fig. 8. Frequency response comparison of 2 in 1 exciter and ellipse type exciter

The demographic characteristics of the respondents who responded to the survey are as follows. The average age of the respondents was 21.97, 23 under 20 years old, 122 in 20 - 25 years old, 14 in 26 - 30 years old. The distribution of gender was similar in $51.6 \%$ of males and $48.4 \%$ of females. Table 1 is as follows.

Table 1. Demographics.

\begin{tabular}{|c|c|c|c|c|c|c|c|}
\hline & & Frequency & $\%$ & & & Frequency & $\%$ \\
\hline \multirow{3}{*}{ Age } & under 20 & 23 & 14.4 & \multirow{2}{*}{ Gender } & male & 82 & 51.6 \\
\hline & $20-25$ & 122 & 76.7 & & female & 77 & 48.4 \\
\hline & $26-30$ & 14 & 8.9 & & & & \\
\hline
\end{tabular}

To verify the inertia and reliability of the measurement tool, exploratory factor analysis, and reliability analysis were performed. The principal components analysis was performed on the exploratory factor. The criterion of eigenvalue was set above 1.0, and the criterion of factor loading was set above 0.4 . As a result of the analysis, sufficient inertia was verified as a measuring tool. The Cronbach's alpha value of the reliability analysis was set to 0.6 or more. The analysis shows that high reliability is obtained. Table 2 shows the results of the factor analysis for the improved sound. 
Table 2. Exploratory and confirmatory factor analysis, reliability, mean, standard deviation, correlations.

Exploratory and confirmatory factor

\begin{tabular}{c|c|c}
\multicolumn{2}{c|}{ Exploratory and confirmatory factor } & reliability \\
$\mathrm{KMO}=.738$ & Component & Cronbach's a \\
\hline intelligibility1 & .879 & \multirow{2}{*}{.873} \\
\hline intelligibility2 & .896 & \\
\hline intelligibility3 & .904 & \\
\hline Eigenvalue & 2.394 & - \\
\hline \% Variance & 79.814 & \\
\hline Cumulative\% & 79.814 & \\
\hline
\end{tabular}

T-test analysis was conducted to analyze the difference in sound quality between the two TVs. In the T-test analysis, when the t value is above 1.96 or below -1.96 , and the significant value is less than 0.1 , the mean is judged to be different under the statistical significance level. As a result, $t$ was -8.684 , less than -1.96 , and significant was .000 , less than .1 . So the sound quality of the two TVs is different, and the previous TV sound is 3.860 on average, and the improved TV sound is 4.528, which means that the sound of the improved TV is high. These results mean that people perceive that the sound quality of an improved TV is better. Table- 4 is a table of the analysis results.

Table 3. t-test analysis

\begin{tabular}{c|c|c|c|c|c}
\hline & TV type & Average & Std. Dev. & t & significant \\
\hline \multirow{2}{*}{ intelligibility } & Old & 3.860 & .813 & \multirow{2}{*}{-8.684} & .000 \\
\cline { 2 - 4 } & Proposed & 4.528 & .636 & & .000 \\
\hline
\end{tabular}

In short, the sound is improved by using a single-core stimulator. The assessment confirmed that the sample applied with the ellipse-type single-core exciter has a better overall balance and improved intelligibility of the mid-tone. It was confirmed that the resolution and openness of the treble was significantly improved compared to the 2-in-1. In addition, it was confirmed that the difference in sound quality according to the left and right position is almost felt, and the left and right sound fields are wider than before.

The dynamic ranges of the two exciters were also tested. The input power to the exciter was checked from $1 \mathrm{~W}$ to $20 \mathrm{~W}$. The results show that the 2 -in- 1 exciter tends to saturate at around $10 \mathrm{~W}$, but the ellipse-type single-core exciter increases linearly to $20 \mathrm{~W}$. Fig. 9 and 10 show the dynamic range of the output of the two types of exciters at $1 \mathrm{~W}, 5 \mathrm{~W}, 10 \mathrm{~W}, 15 \mathrm{~W}$, and 20 $\mathrm{W}$, and the frequency response of the sound pressure generated at that time. Fig. 9 shows the result of the 2-in-1, and it can be seen that the larger the input signal, the higher the output sound pressure. As a result of the speaker's magnetic-field saturation, the sound pressure increase is small from the $10 \mathrm{~W}$ test. Fig. 9 shows the result of a single-core ellipse; although the increase decreases to $20 \mathrm{~W}$, the volume continues to increase. Comparing the results of Fig. 9 and 10, it is confirmed that the proposed ellipse-type exciter is improved over the existing 2-in-1 type in terms of sound pressure and dynamic range. 


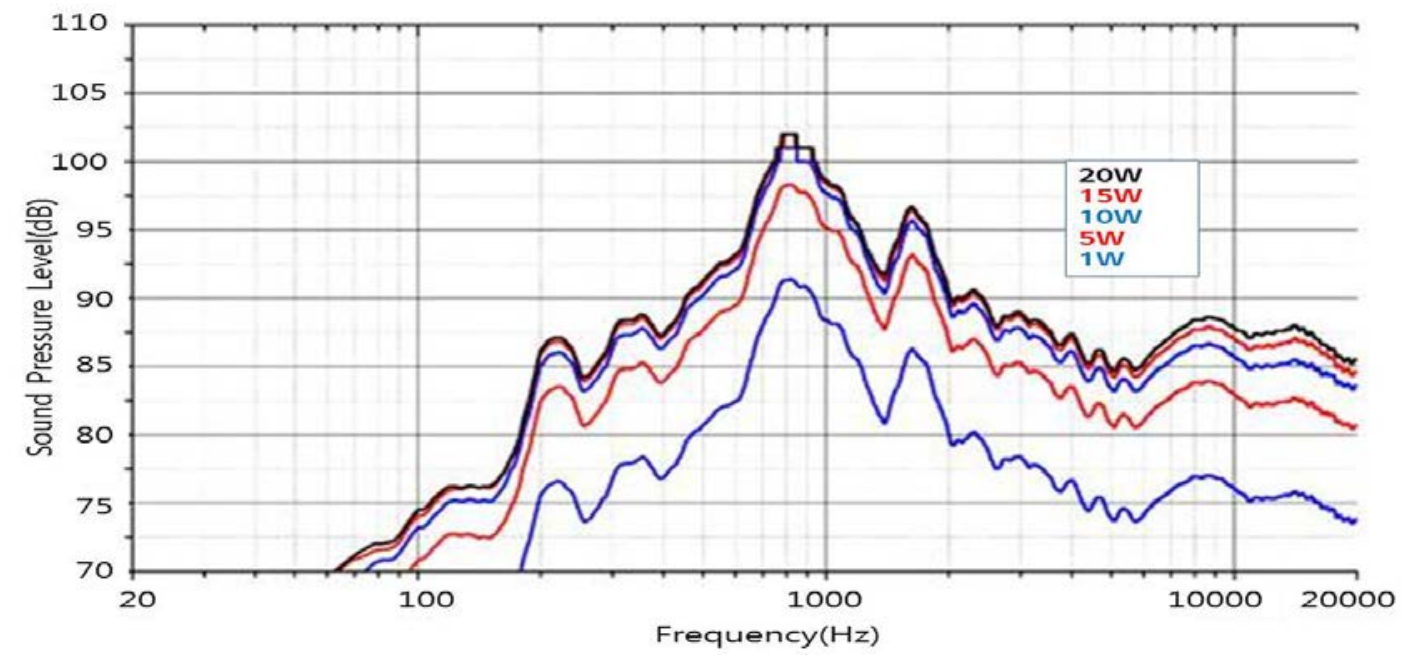

Fig. 9. Dynamic Range Measurement result of 2 in 1 Exciter

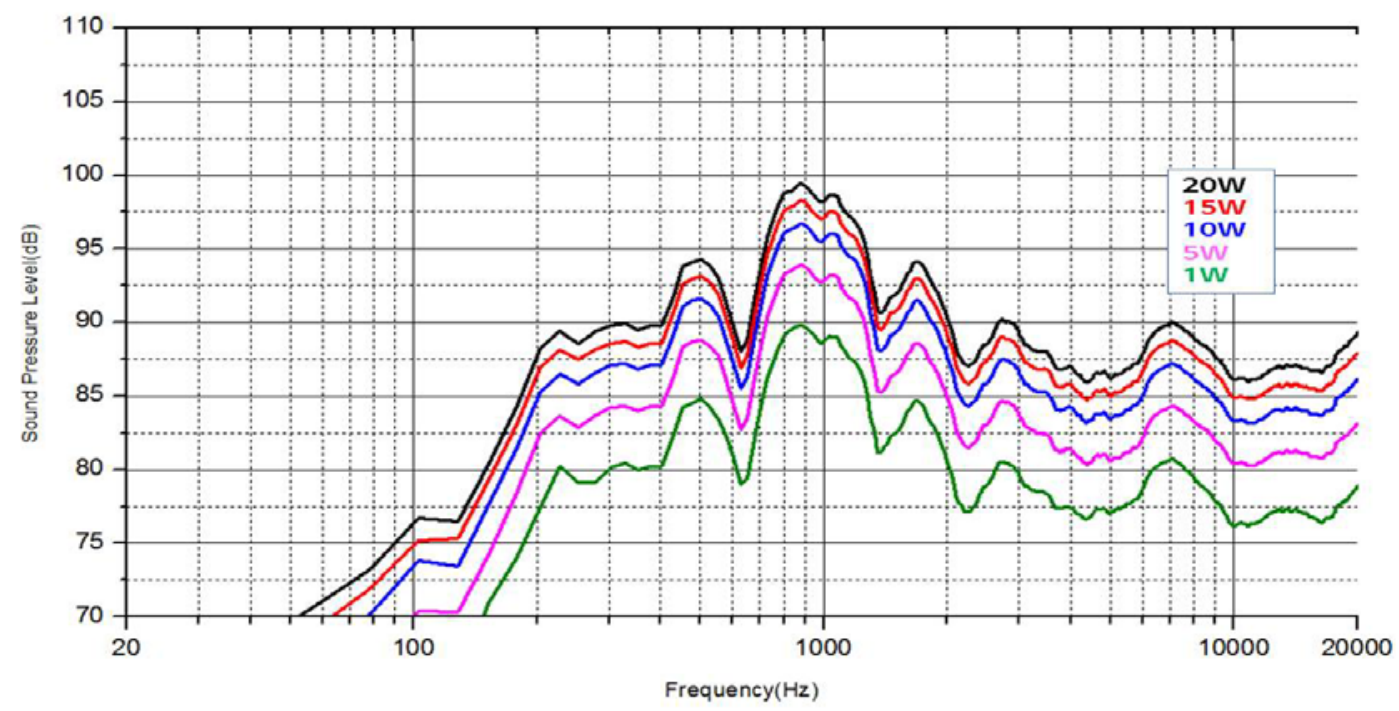

Fig. 10. Dynamic Range Measurement result of Ellipse Type Single Core Exciter

\section{Experiment and result}

\subsection{Directive Characteristic Measurement Experiment}

By applying the Ellipse-Type Single-Core Exciter, the sound quality and dynamic range are improved, and the directivity is also improved compared to the 2-in-1 method. To construct the experiment and measure and analyze the directivity as shown in Fig. 11, we analyzed with B \& K Pulse equipment. Measurement experiments were conducted in an anechoic chamber. In order to confirm the directivity characteristics, the study was conducted by measuring at the angle of the front face and the angle of 30 degrees. 


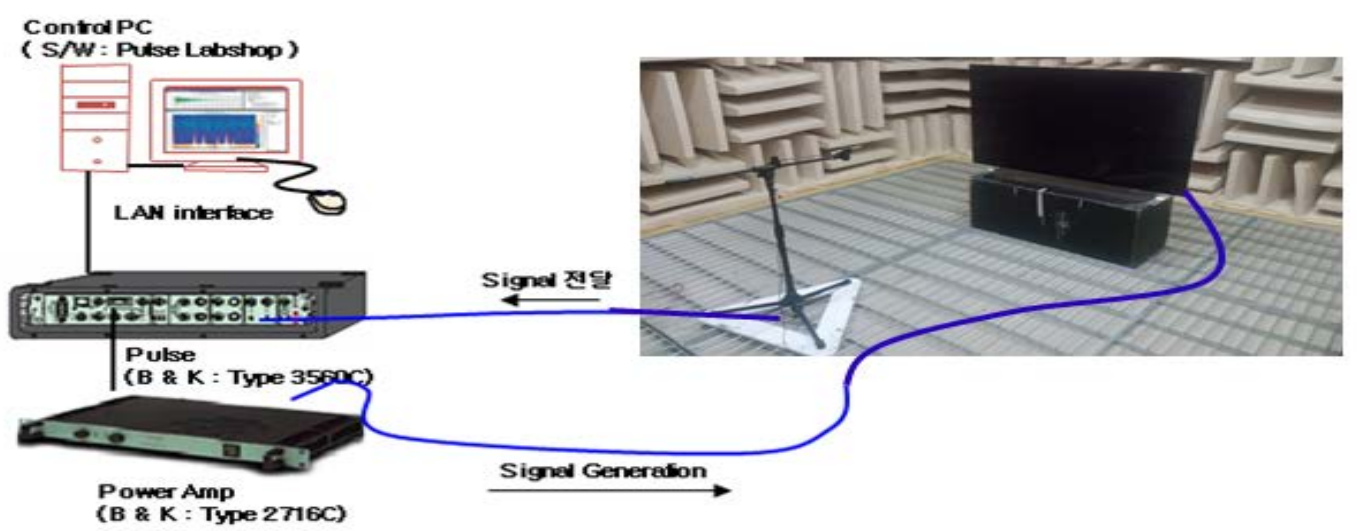

Fig. 11. Directional characteristics Measurement Method

\subsection{Directive property improvement}

In TV speaker systems, the directivity is also an important factor. It is desirable to design a wider range of sweet spots by adjusting the directivity of the acoustic system. In order to do so, a system using a general dynamic speaker may adjust the shape of the enclosure, the contour of the diaphragm of the speaker, the position of the plurality of speaker units, and the beam pattern. It also uses multiple speakers arranged to increase or shift the range of sweet spots. However, it is difficult to apply this acoustic technology in general flat-panel TVs, in which it is very difficult to control sweet spots because of the small, thin, limited sound emission method. Moreover, the position of the speaker is at the rear of the screen. OLED TVs using the proposed 2-in-1 exciter speaker are also intended to improve the directivity characteristics to widen these sweet spots. Thus, in this study, the directivity of the exciter was improved to produce a flattened sound pressure distribution over a wider range. The proposed method proposes a new shape of the vibration system like a single-core exciter speaker and measures and compares the acoustic characteristics. Fig. 12 shows the measurement results of the radiation characteristics of a 2-in-1 exciter speaker. It is the result of measurement of left and right at an angle of 30 degrees based on the front measurement result (black). As can be seen from the results, the side measurement shows that the frequency response is lower in the band above $5 \mathrm{kHz}$, which means that the radiation characteristic of the 2-in-1 exciter is formed in a pointed shape concerning the front side. In particular, $15 \mathrm{~dB}$ is attenuated in the $10 \mathrm{kHz}$ band. For the 2-in-1, the tone of the sound is bright on the front of the Exciter, as has been confirmed by this measurement, and there is a need for improvement. 


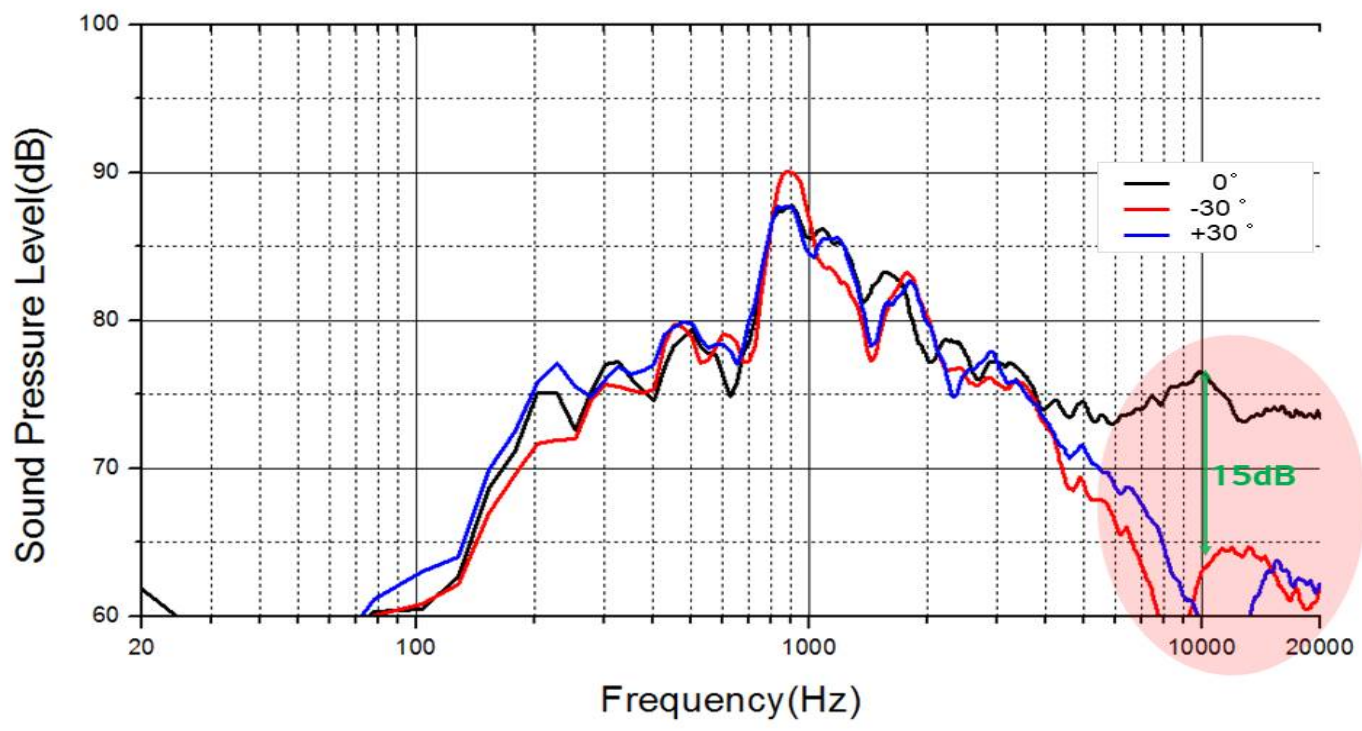

Fig. 12. Directional Characteristics of 2in1 Exciter Speaker

Fig. 13 shows the theoretical considerations that the difference in the delay time of the sound source arises because of the different distances of the transmission path by the two sound sources on the same line, which cause mutually constructive / offset interference on the two cores. Depending on the direction in which the sound source is radiated, the two sound sources cause mutual interference. In addition, the delay time depends on the distance, that is, the speed of the sound path according to the radiated angle, and the sound pressure is affected by the equation shown in the figure.
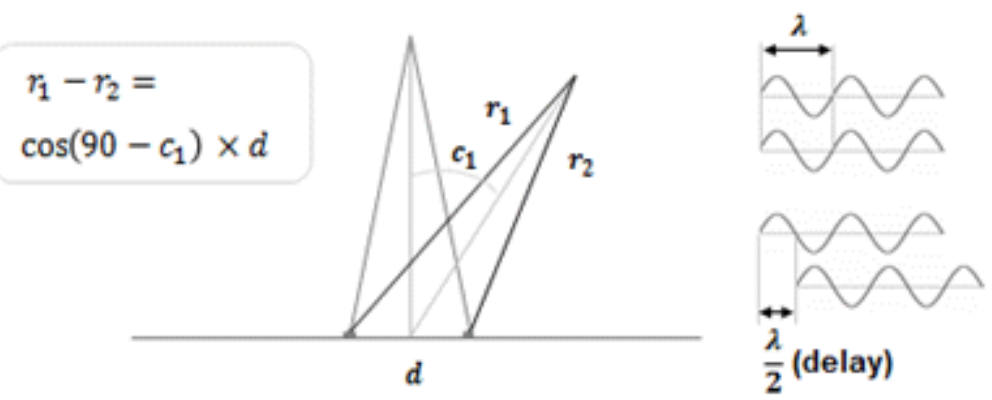

$$
\begin{aligned}
& r_{1}-r_{2}=0+n \lambda \\
& r_{1}-r_{2}=\frac{\lambda}{2}+n \lambda
\end{aligned}
$$

Fig. 13. Effect of radiation angle due to mutual interference of two sound sources [10]

Following the above description, we confirmed that the change is caused by Equation (2) according to the frequency generated in each sound source. In Equation (2), ' $\mathrm{C}$ ' is $340 \mathrm{~m} / \mathrm{s}$ at the speed of sound. 'd' is the distance between the two sound sources, and 'c1' is the angle to be emitted or the angle to be measured [10]. 


$$
f=c /\left(\cos \left(90-c_{1}\right) \times d\right)
$$

Fig. 14 shows measurement results for various beam patterns according to the distance between two excimers at $5 \mathrm{kHz}$. If the distance of the sound source is $100 \mathrm{~mm}$, it can be seen that the dip occurs in the left and right at 20 degrees, and a peak occurs near 40 degrees. If the distance is 200, peaks and dips appear. However, the amount is reduced, and the ripple in the radiation pattern occur at more diverse angles.

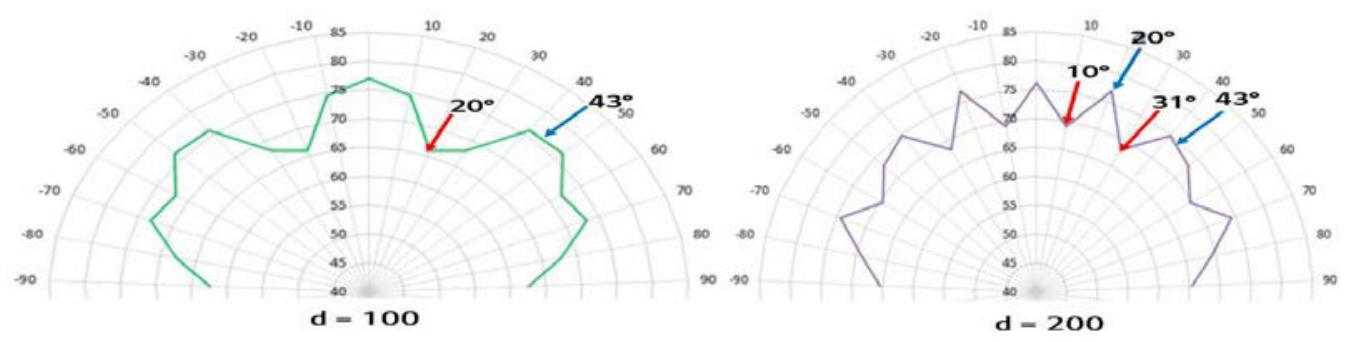

Fig. 14. Measurement Result of exciter beam pattern by distance and radiation angle

Fig. 15 shows the structure of the existing 2-in-1 Exciter. It can be estimated that the dip will occur at a certain angle and frequency because of the distance difference and mutual interference between the exciters E1 and E2. The distance between $1 \mathrm{~cm}$ and $4 \mathrm{~cm}$, which is the closest distance between $\mathrm{E} 1$ and E2, can be expected to dip at $34 \mathrm{kHz}$ and the distance at $8.5 \mathrm{kHz}$. Predictions can be compared with frontal measurements in the band above $5 \mathrm{kHz}$, the measurement results in Fig. 12. With the results, it can be explained that the frequency characteristic value at 30 degrees is attenuated and $15 \mathrm{~dB}$ is attenuated in the $10 \mathrm{kHz}$ band.

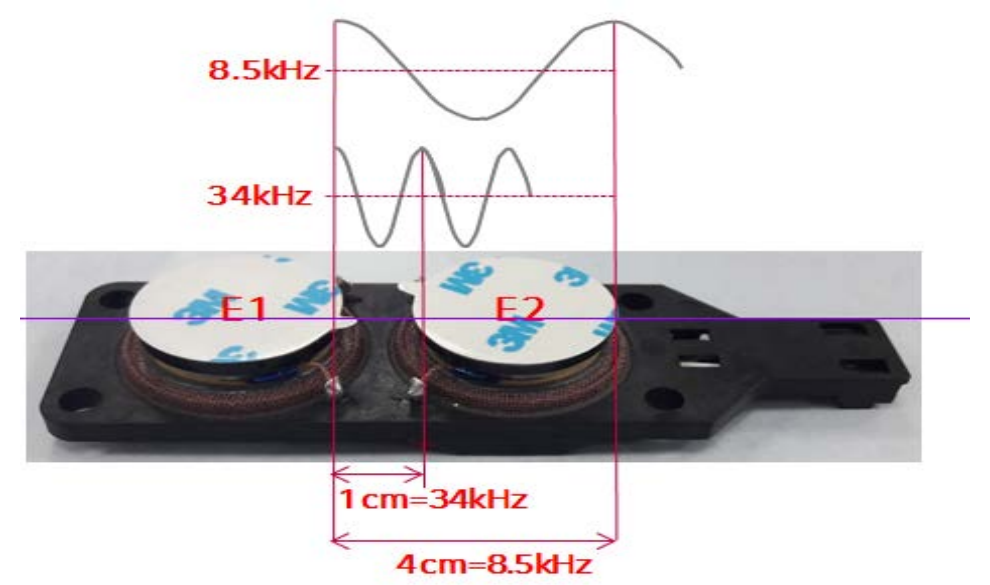

Fig. 15. 2in1 exciter model and attenuation waveform prediction model according to numerical value 
We confirmed that the distance between the two cores in the 30-degree angle direction showed characteristics similar to that of $50 \mathrm{~mm}$ at $40 \mathrm{~mm}$. In order to check the vibration pattern of the panel, we used the laser Doppler measurement to test the interference between the 2 cores per frequency. Interference between the two cores at $5 \mathrm{kHz}$ is destructive interference. Fig. 16 shows the results of LDV measurement, and depending on the interference fringe, it can be confirmed whether constructive interference or destructive interference occurs at a frequency. The part indicated by the arrow shows the destructive interference caused by the reverse phase between the two cores.

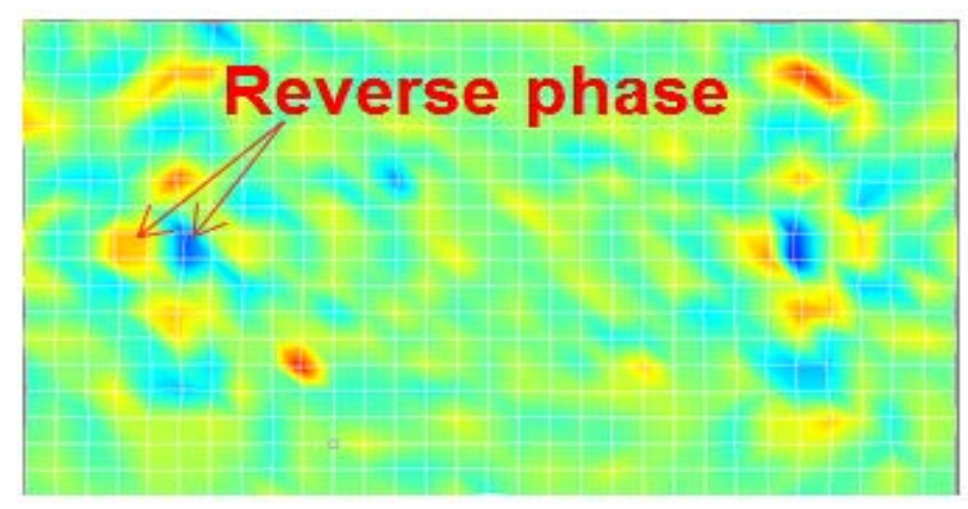

Fig. 16. Interference fringe results from laser doppler measurements

In an exciter speaker, when two cores are used, neither core has consistent behavior, contrary to design intent. Therefore, the cores must be controlled individually in consideration of the size of the two cores, the frequency used, and mutual interference according to the sound-source distance. However, the calculation for the control and the configuration of the independent amplifier of the control is expensive. Therefore, the problem is solved by changing the shape of the core. The improvement is to implement a sound-generating vibration system from the 2-in-1 to a single core. The shape of the Exciter implemented as a single core is elliptical, as shown in Fig. 17. The magnitude of the required magnetic field of the loudspeaker's magnetic system is shown in Fig. 6. In general, excitation is generated at the output of the exciter speaker, and it is designed to be used in a linear range of magnetic.

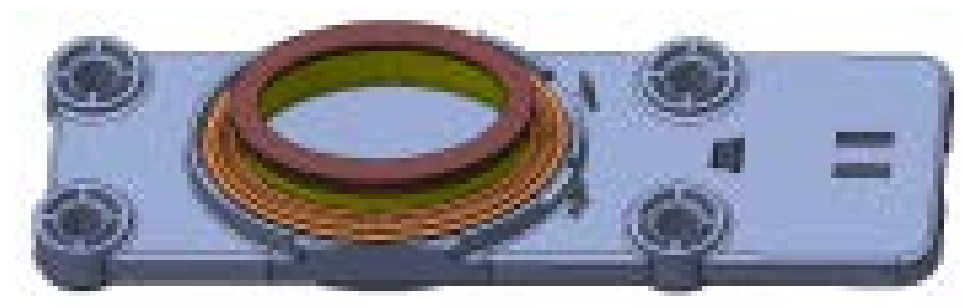

Fig. 17. Design Model of Ellipse Type Single Core Exciter

In the previous 2-in-1 Exciter, the offset interference started at $5 \mathrm{kHz}$, resulting in sound-pressure attenuation. However, when the Ellipse-Type Single-Core Exciter is applied, it can eliminate the influence of mutual interference. In addition, we confirmed that the 
equivalent sound pressure is obtained even at 30 degrees left and right up to $5 \mathrm{kHz}$. Fig. 18 is a graph of frequency characteristics measured at 30 degrees in front, left and right by applying the Ellipse-Type Single-Core Exciter. The difference in the characteristics of the bass is small, and no sound pressure attenuation occurs until $8 \mathrm{kHz}$, where the attenuation is reduced from the existing $15 \mathrm{~dB}$ to $7 \mathrm{~dB}$ at $10 \mathrm{kHz}$. This result means that the sound-pressure radiation pattern of the exciter speaker is widened and that the same number of exciters can create a wide range of sweet spots.

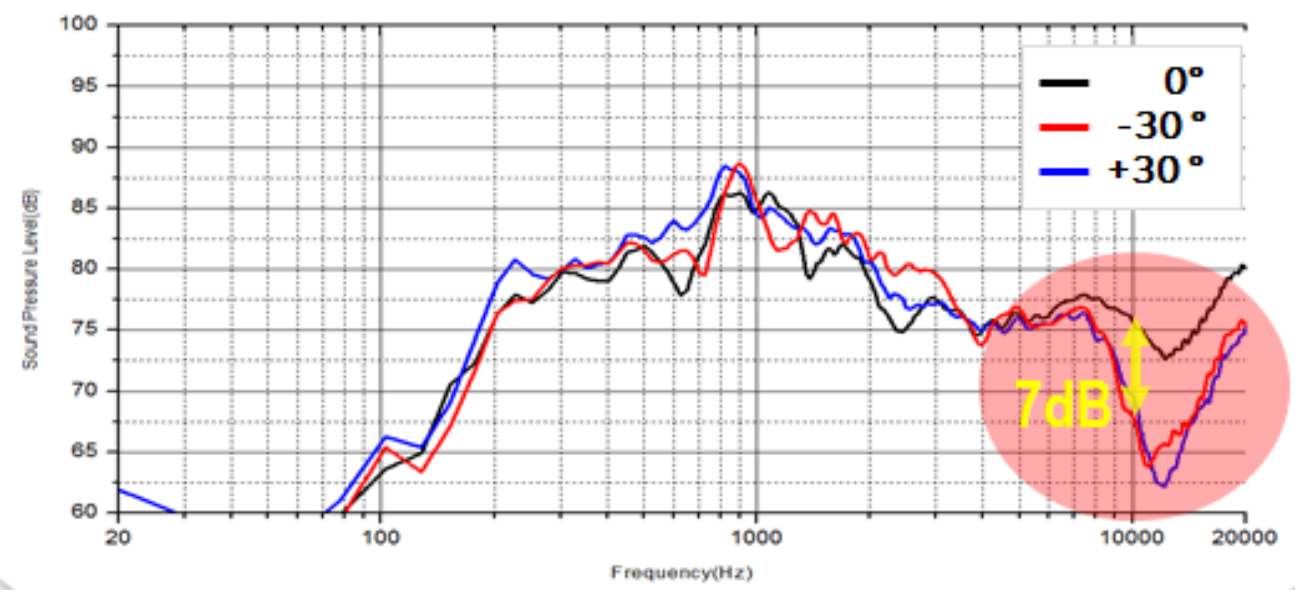

Fig. 18. Frequency response measurement result by azimuth angle of Ellipse Type Single Core Exciter

\section{Conclusion}

Nowadays, display technology has been making great progress day by day. In particular, TVs are released with $8 \mathrm{~K}$, four times the UHD picture quality, boasting the world's best picture quality in terms of clarity. However, the audio system, one of the key TV features, is relatively slower in technology than slim design and high-resolution picture quality. To fit in a slim design, the speakers become thinner and the placement is hidden behind the panel. As the speaker becomes thinner, the acoustic characteristics deteriorate, and the arrangement is also radiated downward, so that the viewer hears the deformed sound reflected from the floor. As a result, the sound quality deteriorates according to the furniture arrangement, the material, and the structure of the lower part, and the image and sound are recognized as separated, thereby reducing the immersion feeling. In our previous study, we proposed an exciter speaker to overcome this shortcoming and compared the sound quality. The improved model of the OLED panel speaker proposed in this paper can construct a wide range of sweet spots by implementing a wide pattern of sound emitted from the panel. The propose method can create a realistic three-dimensional sound because the wide sound field filled the screen. In addition, we introduced a method to improve the sound quality by applying the Ellipse-Type Single-Core exciter. In the proposal we are improving the directivity, which is a disadvantage of the 2-in-1 exciter used in the previous study. The acoustic emission characteristics were improved by $7 \mathrm{~dB}$ (@ $8 \mathrm{kHz}$ ) at the left and right angles of 30 degrees from the front of the Exciter. In the OLED panel, the exciter speaker is the only existing way to maximize the quality and immersion of sound quality in flat-panel TVs. 
In the future, we intend to manufacture a multi-channel OLED Panel exciter speaker equipped with two exciters with two left and right channels, as well as a study that can steer the sound in a specific direction. We will study various applications by applying 3D sound technology.

\section{References}

[1] Republic of Korea OLED Yesterday, Today and Tomorrow, Korea Display Industry Association, Sainovation, 2016.

[2] S. T. Lee, H. W. Park, M. J. Bae and K. H. Park, "Sound Quality Improvement of a Flat-Panel Display Front Speaker(Emotional and EEG Test of Exciter Speaker)," SID Symposium Digest of Technical Papers, Vol. 48, Issue 1, pp. 1680-1681, 2017. Article (CrossRef Link)

[3] “AMOLED Industry Market Tracker”, IHS Report, 2017 2018.

[4] H.J. Shin, M.Y. Son, B.H. Kim, Y.H. Kim, C,A, Lee, K.S. Kim, J.H. Choi, J.J. Kim, C.H. Oh, and I.B. Kang, "A Novel High Speed Integrated Gate Driver Circuit using a-Si TFT for 240Hz FHD LCD TVs,” SID Symposium Digest of Technical Papers, Vol. 42. No. 1, pp.186-189, 2011. Article (CrossRef Link)

[5] W.J. Nam, B.C. Ahn, S.Y. Cha and S.D. Yeo, "Technological Progress and Commercialization of OLED TV," SID Symposium Digest of Technical Papers, Vol. 44. No. 1. Oxford, UK: Blackwell Publishing, 21-1, 2013. Article (CrossRef Link)

[6] S.T. Lee, K.H. Park, K.G. Jang, and C.H. Oh, "Study on Enhancement of the Sound Quality by Improvement of Panel Vibration in OLED TV," SID Symposium Digest of Technical Papers, Vol. 49. No. 1. 2018. Article (CrossRef Link)

[7] Hiroyuki Takewa, Shuji Saiki, Sawako Kano and Atsushi Inaba, "Slim-type speaker for flat-panel televisions,” IEEE Transactions on Consumer Electronics, Vol. 52, No. 1, pp. 189-195, 2006. Article (CrossRef Link)

[8] S. Lee, Basic Properties of Sound and Application, Chung-Moon-Gak Publisher, 2004.

[9] S.J. Oh, Theory and design of loudspeaker, SuckHakDnag press, 2011.

[10] S. Lee, H. Park, K. Park, and M. Bae, "A study on the optimal speaker position for improving sound quality of flat panel display," The Journal of the Acoustical Society of America, Vol. 144, No. 3, 2018. Article (CrossRef Link)

[11] J. T. Kim, J. H. Kim, J.O. Kim and J.K. Min, "Acoustic Characteristics of a Loudspeaker Obtained by Vibroacoustic Analysis," Trans. Korean Soc. Mech. Engineering, Vol. A21, No.10, pp. 1742-1756, 1997.

[12] H.W. Park and M.J. Bae, "A Study on the Improvement of Sound Quality According to the Location of OLED Flat Plate Speaker," Asia-pacific Journal of Multimedia Services Convergent with Art, Humanities, and Sociology, Vol.7, No.12, pp. 775-783, 2017.

[13] S. Lee, H. Park, M. Bae, and K. Park, "Study on multi-channel speakers for controlling sound field of flat panel display,” The Journal of the Acoustical Society of America, Vol 143, No.3, 2018. Article (CrossRef Link)

[14] J.H. Kim, J.W. Kim, “Thin speakers and transparent speakers,” CERAMIST, Vol. 17, No. 2, pp.60-66, 2014. Article (CrossRef Link)

[15] H. J. Beon, "Trends in speaker manufacturing technology at domestic and abroad," The Magazine of the IEEE Korea, Vol. 13, No.6, pp.513-520, 1986.

[16] Jeremy C. Franklin, Fletcher R. Rothkopf, Scott A. Myers, and Stephen Brian Lynch, "Display-based speaker structures for electronic devices," U.S. Patent, No. 8,934,228. 13 Jan. 2015.

[17] D.J. Choi, Y. W. Park, H. J. Park, "Research of Relation Between Sound Pressure and Magnetostrictive Speaker For 2ch Flat Display,” in Proc. of Korean Society for Precision Engineering, 2010 Fall Conference, pp.603-604, 2010.

[18] H. W. Choi, Y. J. Kim, Y. W. Park, "Acoustic analysis and vibration modeling for design of flat vibration speaker," in Proc. of Korean Society for Precision Engineering, pp. 545-546, 2008. 
[19] P. Sun, J.H. Park, J. Kwon, and S. Hwang, "Development of Slim Speaker for Use in Flat TVs," IEEE TRANSACTIONS ON MAGNETICS, VOL. 48, NO. 11, 2012. Article (CrossRef Link)

[20] W. Hoeg, L. Christensen, and R. Walker, "Subjective assessment of audio quality-the means and methods in the EBU,” EBU Technical Review, pp. 40-50, 1997.

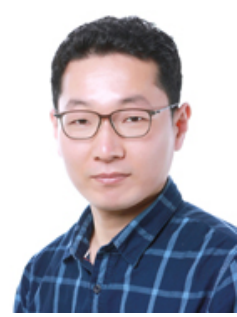

Sungtae Lee is a OLED TV Future Development Research Engineer of the LG Display, Seoul, Korea. His current research interest includes OLED TV Panel, Speaker Tech, Audio Signal Processing, Actuator Technology, OLED Panel Speaker, Future OLED Display, Multi channel Speaker System, and Audio Amplifier.

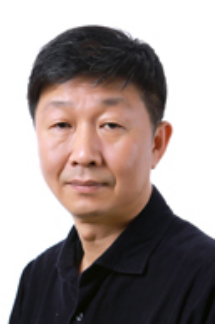

Kwanho Park is a OLED TV Future Development Research Fellow of the LG Display, Seoul, Korea. His current research interest includes OLED TV Panel, Commercial Display, Audio Signal Processing, OLED Compensation Circuit, Actuator Technology, OLED Panel Speaker, Future OLED Display, Multi channel Speaker System, Audio Amplifier, Rollable \& Transparent Display Mechanic Tech.

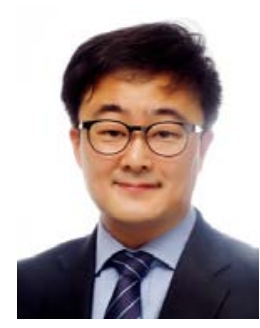

Hyung Woo Park received a Ph.D., an M.S., and a B.S. in Electrical Engineering from Soongsil University. He is an assistant professor at the Information and Technology Department at Soongsil University, Seoul, Korea. His current research interest includes sound signal processing, big data analysis, voice analysis, noise reduction system, wave field synthesis, railway noise, and Internet of Things. 